\title{
Face Recognition Using Local Binary Decisions
}

\author{
Alex Pappachen James and Sima Dimitrijev
}

\begin{abstract}
The human brain exhibits robustness against natural variability occurring in face images, yet the commonly attempted algorithms for face recognition are not modular and do not apply the principle of binary decisions made by the firing of neurons. We present a biologically inspired modular unit implemented as an algorithm for face recognition that applies pixel-wise local binary decisions on similarity of spatial-intensity change features. The results obtained with a single gallery image per person show a robust and high recognition performance: $94 \%$ on AR, $98 \%$ on Yale, $97 \%$ on ORL, $97 \%$ on FERET (fb), $92 \%$ on FERET (fc), and $96 \%$ on Caltech face image databases.
\end{abstract}

Index Terms-Image edge analysis, threshold logic.

\section{INTRODUCTION}

$\mathbf{T}$ HE fundamental problem that hinders the development of a robust automatic face recognition system is the natural variability that occurs in face images. Major methods that are employed to reduce this problem can be classified into three groups: 1) methods whose so-called gallery set consists of multiple training images per person [1], [2], 2) image preprocessing techniques that aim at feature restoration [3], and 3) use of geometrical transforms to form face models [3]. Even though they show high performance under specific conditions, they lack overall robust performance and in many cases have proved to be computationally expensive. As distinct from these computational schemes, the human visual system, which is the best available natural model for face recognition, uses modular approach for classification of faces [4]. In this letter, we present a method that implements the concept of local binary decisions to form a modular unit and a modular system for face recognition. We apply the method to formulate a simple algorithm, and we verify its robustness against natural variability occurring in face images.

\section{LOCAL BINARY DECISIONS}

Binary decisions (or yes/no decisions) are inevitable in any classification task. In the case of the human brain, these decisions are made by the firing of its neurons, which means that they are local binary decisions. Most existing face recognition approaches utilize probabilistic or deterministic formulations to

Manuscript received July 25, 2008; revised August 28, 2008. This work was supported by the Australian Research Council. The associate editor coordinating the review of this manuscript and approving it for publication was Dr. Vince D. Calhoun.

The authors are with the Queensland Microtechnology Facility and Griffith School of Engineering, Griffith University, Nathan 4111, Australia (e-mail: apj@ieee.org).

Color versions of one or more of the figures in this paper are available online at http://ieeexplore.ieee.org.

Digital Object Identifier 10.1109/LSP.2008.2006339
TABLE I

NORMALIZED SIMILARITY MEASURES FOR THE CLASSIFIER

\begin{tabular}{|l||l|}
\hline Type & Equation $^{\mathrm{a}}$ \\
\hline Min-max ratio & $\min \left[x_{g}, x_{t}\right] / \max \left[x_{g}, x_{t}\right]$ \\
Difference & $\left.\mid x_{g}-x_{t}\right) \mid / \alpha$ \\
Exponential difference & $e^{-\left|x_{g}-x_{t}\right| / \alpha}$ \\
& where $\alpha$ is $\max \left[x_{g}, x_{t}\right]$ or \\
& {$\left[x_{g}+x_{t}\right] / 2$ or $\min \left[x_{g}, x_{t}\right.$} \\
\hline \\
\multicolumn{2}{|c}{ The vector multiplications and divisions are element by element operations. It } \\
can be also noted that choice of $\alpha$ do not alter the recognition performance.
\end{tabular}

delay the binary decisions until a global level is reached. However, it is well established in digital electronics that local binary decisions can remove noise in a way that is not possible in analog systems, regardless of whether probabilistic or deterministic formulations are used in the analog system. Inspired by these analogies, we apply local binary decisions to every pixel of the images that are being compared.

In addition, the binary decisions necessitate the use of a threshold $\theta$ so that some differences can be ignored as the decisions on similarities are made. The most common method for similarity matching is the use of various distance measures [5]. The similarity measures need to be normalized to enable their use with a global threshold. A normalized comparison between two feature vectors $x_{g}$ and $x_{t}$, formed from the gallery and test images, respectively, can be achieved in many ways. The simplest way to form a normalized measure $\delta_{g t}$ is by taking an element-by-element ratio of the minimum and the maximum of the vectors $x_{g}$ and $x_{t}$ (Table I). Other possibilities, also shown in Table I, include normalized difference and exponential difference. Thresholding the similarity measure results in a binary vector representing the local decisions made by the element-wise comparisons. Taking the sum of all the elements in this vector forms the global similarity score for the two variables. Following the comparisons of the test image to all the gallery images, we rank the similarity scores. This completes the steps of what is commonly referred to as a classifier.

\section{SPATIAL INTENSITY CHANGES}

The raw image vector, which could be the simplest feature vector, is highly sensitive to variation in illumination. This problem can be reduced by using spatial intensity changes as the feature vector. Furthermore, a number of published results suggest that the spatial intensity change is the essential visual cue required for recognition [6]. There are many ways of calculating the spatial intensity changes from a raw image vector. In this letter, vectors of spatial intensity changes $\sigma_{g}$ from gallery image $I_{g}$ and $\sigma_{t}$ from test image $I_{t}$ are calculated by using any of the following standard local spatial filters: ${ }^{1}$ standard deviation, range [7], and gradient [8]. As an example, the spatial

\footnotetext{
${ }^{1}$ These filters are available in MATLAB 7 as a part of the image processing library, whose function names are: stdfilt, rangefilt, and gradient.
} 


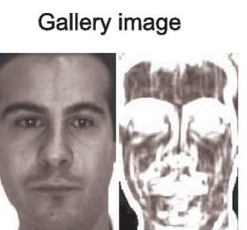

(a)

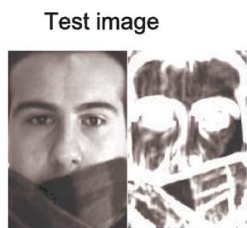

(c) (d)
Gallery image

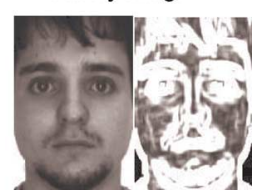

(e) (1) (b)

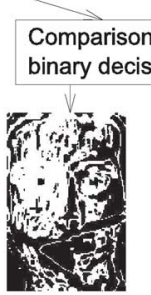

(g)
Local binary decision vectors

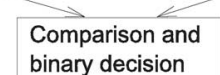

binary decision

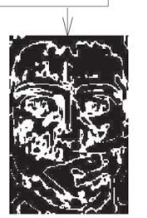

(h)
Fig. 1. Graphical overview of various components in the modular unit using images from AR face database [9]. The face images labeled by (a) and (e) represent sample face images with neutral expressions, which are selected as the gallery set. The images labeled by (b) and (f) are the feature vectors of images (a) and (e), respectively, obtained as the normalized spatial-intensity change features using a local standard deviation filter [using (1)] followed by local mean normalization of the features [using (2)] . The image labeled by (c) is a sample test image, and the image labeled by (d) is its corresponding feature vector. The image pattern labeled by $(\mathrm{g})$ is the result of binary decisions made by comparing (c) with (a), whereas image pattern (h) is the result obtained by comparing (c) with (e). Clearly, in this case, (a) is the best match to (c) because more positive similarity decisions (white pixels) appear in $(\mathrm{g})$ than in $(\mathrm{h})$.

intensity change $\sigma$ can be calculated using standard deviation filter on each pixel by applying the following equation:

$$
\sigma(i, j)=\sqrt{\frac{1}{m n} \sum_{s=-a}^{a} \sum_{t=-b}^{b}[I(i+s, j+k)-\overline{I(i, j)}]^{2}}
$$

where $a=(m-1) / 2, b=(n-1) / 2$, and $\overline{I(i, j)}$ is the local mean of image pixels intensities in a window of $m \times n$ pixels surrounding the pixel located at $(i, j)$. Further, to obtain the feature vectors $x_{g}$ and $x_{t}$, normalizations are performed by dividing each element of $\sigma_{g}$ and $\sigma_{t}$ by their regional means, $\overline{\sigma_{g}}$ and $\overline{\sigma_{t}}$, calculated using centered local windows of constant size.

\section{Modular Unit AND Modular System}

The classifier, based on the local binary decisions on similarity and the spatial intensity change filter, form the modular unit for face recognition. A graphical overview of the modular unit is shown in Fig. 1. To demonstrate the use of this modular unit in more complex systems, we form a modular system and apply it to 1) color images and/or 2) shifted, rotated, and/or scaled images to minimize localization errors.

\section{LOCAL BINARY DECISIONS ON SIMILARITY ALGORITHM}

The following four steps summarize the local binary decisions on similarity (LBDS) algorithm that is used in this letter to present and analyze the new modular unit and a related modular system.

1) The spatial intensity change features $\sigma_{g}$ and $\sigma_{t}$ are calculated by using any of the standard local spatial filters: standard deviation [see (1)], range [7], gradient [8]. The normalized spatial intensity changes are determined by the following equations: ${ }^{2}$

$$
\begin{aligned}
& x_{g}(i, j)=\frac{\sigma_{g}(i, j)}{\left[\overline{\sigma_{g}}(i, j)+\epsilon\right]} \\
& x_{t}(i, j)=\frac{\sigma_{t}(i, j)}{\left[\overline{\sigma_{t}}(i, j)+\epsilon\right]} .
\end{aligned}
$$

2) The normalized ratio $\delta_{g t}$ between the two feature vectors $x_{g}$ and $x_{t}$ under comparison is calculated using any of the similarity measures listed in Table I.

3) A global threshold $\theta$ is applied to $\delta_{g t}$, which forms a binary vector. All the elements of the binary vector are summed to form the similarity score $S_{k}{ }^{*}$ between the two compared images: $S_{k}^{*}=\operatorname{Sum}\left\{I\left[\delta_{g t}(i, j)>\theta\right]\right\}$, where $I$ is the binary operator.

a) If color images are used, the similarity scores are calculated for the red $S_{k}^{r}$, green $S_{k}^{g}$, and blue $S_{k}^{b}$ components independently using steps 1 to 3 and $S_{k}^{*}$ for the image is calculated as the maximum of similarity scores obtained as the additive combinations of color components ${ }^{3}$

$$
S_{k}^{*}=\max \left[\left(S_{k}^{r}+S_{k}^{g}\right),\left(S_{k}^{r}+S_{k}^{b}\right),\left(S_{k}^{g}+S_{k}^{b}\right)\right] .
$$

b) To compensate for localization errors, the test image is modified $K$ times by applying shifts, rotations, and/or scaling. Every modified image, when compared with a gallery image, results in a similarity score $S_{k}^{*}$ that is calculated using steps 1 to 3 for gray images and steps 1 to 3 a for color images. The final similarity score $S_{g t}$ for the test image with a compared gallery image is found by selecting the maximum $S_{k}^{*}$

$$
S_{g t}=\max \left(S_{1}^{*}, \ldots, S_{k}^{*}, \ldots, S_{K}^{*}\right)
$$

c) Another way to compensate for localization errors is to modify the gallery images by applying shifts, rotations, and/or scaling. Each generated image is treated independently and the similarity score is calculated by using steps 1 to 3 for gray images and steps 1 to 3 a for color images. Again, the final similarity score $S_{g t}$ is found by selecting the maximum $S_{k}^{*}$ [same as (5)].

4) The final similarity scores corresponding to each gallery image are ranked

$$
P^{*}=\arg _{g} \max S_{g t} .
$$

${ }^{2} \mathrm{~A}$ small constant value $\epsilon=1 \times 10^{-16}$ is used to avoid numerical errors during the computation.

${ }^{3}$ It is known that opponent color is a feature of normal human color processing, which produces luminance channel (red+green) and two chromatic channels (red-green and red+green-blue). However, it is not known how color perception is done. Assuming that each color component contributes equally, makes it logical to try the additive combinations of the channels. Further, we find that (4) performs better than other possible linear combinations of red, green, and blue channels. 


\section{EXPERIMENTAL DETAILS}

We apply the LBDS algorithm to AR [9], ORL[10], Yale [11], Caltech [12], and FERET [13] standard face image databases. At any specific time, illumination, occlusions, face expressions, and time gap between the gallery and test images form variabilities that make the face recognition difficult. A difficult and practically important face-recognition task is created by limiting the gallery to a single image per person. Unless otherwise specified, the results presented in this letter are obtained by this kind of open-set testing.

For each image in the AR, Yale, and Caltech databases, the eye coordinates of the face images are registered manually. For FERET database, the eye coordinates provided in the FERET distribution DVD is used for face alignment. The face alignment is done by rotating, shifting, and scaling the faces so that for all the faces the distance between the eyes remains constant and in fixed spatial coordinates. All the images were aligned and cropped to image size of $160 \times 120.4$ Further, ORL images are localized using manual alignment with warping and are resized to a size of $40 \times 32$ pixels.

Since the eye coordinates of the faces in AR, Yale, and Caltech databases are detected manually, they show shift errors after processing. The eye coordinates of the faces in the gray FERET database are provided within the FERET distribution DVD and, when used, show rotation and scaling errors. A modular system can be formed to compensate for these localization errors. To form the modular system, we use steps 1 to 3 of the algorithm with step $3 \mathrm{a}$ to incorporate color and step $3 \mathrm{~b}$ to compensate for the localization errors (only shift errors) in AR, Yale, and Caltech databases. To compensate for rotation and scale errors in the gray FERET database, we use steps 1 to 3 with step $3 \mathrm{c}$ in the algorithm. In either case, the modifications are in the range of 1 to 6 pixels.

Unless otherwise specified, we use the following global settings for the parameters used in the experiments. To calculate spatial intensity change, we use the local standard deviation filter [see (1)] with optimal window size of $7 \times 5$ and $3 \times 3$ pixels when image size is $160 \times 120$ and $40 \times 30$ pixels, respectively. The local mean normalization window size is set at $80 \times 60$ and $20 \times 16$ pixels when image size is $160 \times 120$ pixels and $40 \times 32$ pixels, respectively. We use the min-max similarity ratio shown in Table I. Finally, the value of the global threshold $\theta$ is set to 0.7 which is selected empirically. The value of $K$ representing number of perturbation used for compensating localization errors in every case is set to a value of 18 .

\section{ReSULTS AND DISCUSSION}

The overall recognition accuracy for the 2500 test images and the gallery size of 100 in the AR database is $94 \%$. This very high accuracy level is possible due to the consistent performance over the large number of variable conditions that are individually listed in Table II (the column labeled as Modular system). Similar accuracy levels are obtained for Yale, ORL, and Caltech databases as shown in Table III. As expected, increased

\footnotetext{
${ }^{4}$ This is done using the Unix script provided for face normalization in the CSU Face Identification Evaluation System, Version 5.0 [14].
}

TABLE II

SUMMARY OF THE RESULTS ON AR DATABASE

\begin{tabular}{|c|c|c|c|c|}
\hline \multirow{3}{*}{ Condition index ${ }^{b}$} & \multicolumn{4}{|c|}{ Top rank recognition accuracy $(\%)$} \\
\hline & \multicolumn{2}{|c|}{ Modular unit $^{\mathrm{a}}$} & \multicolumn{2}{|c|}{ Modular system $^{a}$} \\
\hline & $\mathrm{S} 1^{\mathrm{c}}$ & $\mathrm{S} 2^{\mathrm{c}}$ & $\mathrm{S} 1^{\mathrm{C}}$ & $\mathrm{S} 2^{\mathrm{c}}$ \\
\hline (a) & 100 & 84 & 100 & 98 \\
\hline (b) & 98 & 80 & 99 & 96 \\
\hline (c) & 91 & 72 & 98 & 82 \\
\hline (d) & 94 & 79 & 98 & 80 \\
\hline (e) & 90 & 63 & 96 & 82 \\
\hline (f) & 82 & 56 & 96 & 80 \\
\hline (g) & 100 & 87 & 100 & 95 \\
\hline (h) & 100 & 92 & 100 & 96 \\
\hline (i) & 94 & 59 & 98 & 70 \\
\hline (j) & 99 & 95 & 100 & 100 \\
\hline (k) & 99 & 89 & 100 & 98 \\
\hline (1) & 89 & 68 & 100 & 92 \\
\hline (m) & N/A & 95 & N/A & 100 \\
\hline
\end{tabular}

${ }^{a}$ The baseline results, using the modular unit, are obtained with gray images with no compensation for localization errors. The modular system comprises of modular units applied to the red, green, and blue components of the color images and to modified test images to compensate for localization errors. Single training image per person is used to form the gallery set. The size of the gallery set is 100 and the size of the test sets is 100 for each of the 25 conditions.

b (a) Eye occlusion; (b) Mouth occlusion; (c) Eye occlusion with left illumination; (d) Eye occlusion with right illumination; (e) Mouth occlusion with left illumination; (f) Mouth occlusion with right illumination; (g) Smile; (h) Anger; (i) Scream; (j) Left illumination; (k) Right illumination; (1) Left and right illumination; (m) Neutral

c S1 represents images taken on first day and S2 represents images taken after a time gap of two weeks.

TABLE III

SUMMARY OF THE RESULTS ON DIFFERENT DATABASES

\begin{tabular}{|c|c|c|c|}
\hline \multirow[b]{2}{*}{$\begin{array}{l}\text { Condition } \\
\text { index }^{\mathrm{a}}\end{array}$} & \multirow[b]{2}{*}{ Database $^{b}$} & \multicolumn{2}{|c|}{$\begin{array}{l}\text { Top rank recognition } \\
\text { accuracy }(\%)\end{array}$} \\
\hline & & $\begin{array}{l}\text { Modular } \\
\text { unit }\end{array}$ & $\begin{array}{l}\text { Modular } \\
\text { system }\end{array}$ \\
\hline (a) & Caltech & 91 & 96 \\
\hline (a) & Yale & 94 & 98 \\
\hline (b) & ORL & 92 & 97 \\
\hline (c) & FERET:Fb & 86 & 97 \\
\hline (d) & FERET:Fc & 72 & 92 \\
\hline (e) & FERET:Dup I & 52 & 70 \\
\hline (f) & FERET:Dup II & 40 & 68 \\
\hline
\end{tabular}

a (a) Expression and illumination with a small gallery; (b) Small pose variation on small gallery (c) Expression on large gallery ( $\mathrm{Fb}$ ); (d) Illumination on large gallery $(\mathrm{Fc})$; (e) Large gallery with mean time gap of 251 days (Dup I); (f) Large gallery with mean time gap of 627 days (Dup II)

b Single training image per person is used to form the gallery set. The sizes of Single training image per person is used to form the gallery set. The sizes of
the gallery sets are 28 in Caltech, 15 in Yale, 40 in ORL and 1196 in FERET databases; the sizes of the test sets are 150 in the Yale database, 406 in the Caltech database, 360 in the ORL database, 1194 in set Fb, 194 in set Fc, 722 in Dup I, and 234 in Dup II of the FERET database.

variations correspond to decreased recognition accuracies in all databases. The demonstrated robustness of the algorithm is consistent with the fact that the modular unit does not require any prior knowledge of the specific condition that causes the dominant variations. To substantiate the claim of robustness, it is important to report the performance for a large gallery set. In practice, an increased gallery size decreases the overall recognition accuracy of any face recognition system. The results of testing with the FERET database, also shown in Table III, demonstrate that the robustness is maintained under this condition.

Using the AR database, we analyzed the effects of block size used to make the local binary decisions, and the results are shown in Fig. 2. The maximum recognition accuracy is achieved when the local binary decisions are made at the level of individual pixels (block size of one pixel) with a steep drop in the recognition accuracy as the block size is increased. This directly 


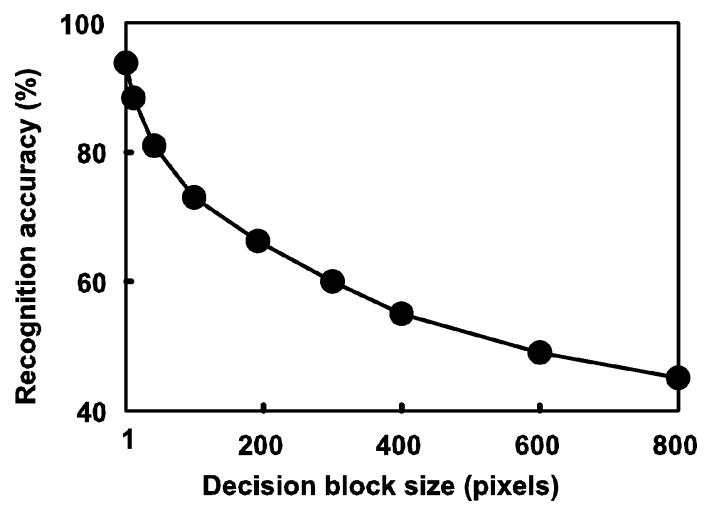

Fig. 2. Dependence of the overall recognition accuracy on the block size used to make the local binary decisions. The resolution of the images is $160 \times 120$ pixels. The window size of the standard-deviation filter is $7 \times 5$ pixels and the size of the normalization window is $80 \times 60$ pixels.

implies that larger image resolutions could further improve the recognition accuracy.

We also analyzed the impact of different implementations of the similarity measure. Using the implementations listed in Table I, the change observed in the recognition accuracy is within $1 \%$. Furthermore, the global threshold $\theta$ for making the local decisions is not a sensitive parameter. We find that the recognition accuracy remains within $1 \%$ across various databases for a range of threshold values from 0.6 to 0.8 . This confirms the general applicability of localized decisions on similarity as a concept.

To analyze the impact of the spatial intensity change in the modular unit, we removed it by using the raw images as the feature vectors. The recognition accuracy for the AR database dropped from $94 \%$ to $63 \%$. Furthermore, we investigated different filters for calculating the spatial intensity changes. The variation of the recognition accuracy with the standard local spatial filters - standard deviation, range, and gradient - is within $1 \%$. Based on this and the clear performance difference between the use of raw images and the spatial intensity changes as the feature vectors, we conclude that the spatial intensity change is the visual cue for face recognition.

The importance of color was also analyzed by comparing the recognition accuracies for color and gray images in the AR database. The accuracy drops from $94 \%$ to $91 \%$ when the color images are replaced by gray images. The impact of test-image shifts, used to compensate for localization errors, is somewhat higher: the overall accuracy drops from $94 \%$ to $89 \%$ when test images without any shift are used.

The overall accuracy for the modular unit (gray images with no shifts) is $86 \%$, which is a very high performance for open-set testing (Table II presents the performance across different conditions). Finally, we tested the recognition performance of the modular unit with multiple images per gallery on the images from the AR database. Using 13 randomly selected images without repetition to form the gallery set, which leaves 13 images per person for the test set, the recognition accuracy increases to $96 \pm 3 \%$.

\section{CONCLUSIONS}

In this letter, we identify local binary decisions as an important concept that is required for recognition of faces under difficult conditions. We also identify spatial intensity changes as the visual cue for face recognition. A modular unit, formed by implementing the local binary decisions for the classifier and the spatial intensity changes for the feature extractor, shows a robust performance under difficult testing conditions. To increase the recognition performance, we formed a modular system by replicating the modular unit. Using this modular system, we analyzed the effect of color, localization errors, and the use of multiple gallery images per person. The analysis shows that the application of the principles of local binary decisions and modularity results in a highly accurate face recognition system. The presented module does not use any known configurational information from the face images, which makes it applicable to any visual pattern classification and recognition problem. Furthermore, classifiers based on the local binary decisions on similarity can be used in other pattern recognition applications.

\section{ACKNOWLEDGMENT}

The authors would like to thank K. Paliwal and Y. Gao of the Institute for Integrated and Intelligent Systems, Griffith University, for useful discussions, for the review of the algorithm, and for reviewing the manuscript. The authors also would like to thank A. Martinez of Computational Biology and Cognitive Science Lab, Ohio State University, for providing access to the AR database.

\section{REFERENCES}

[1] R. Jenkins and A. M. Burton, " $100 \%$ accuracy in automatic face recognition," Science, vol. 319, p. 435, Jan. 2008.

[2] K. Etemad and R. Chellappa, "Discriminant analysis for recognition of human face images," J. Opt. Soc. Amer. A, vol. 14, pp. 1724-1733, 1997.

[3] J. Ahlberg and F. Dornaika, Handbook of Face Recognition. Berlin, Germany: Springer, 2004.

[4] S. Moeller, W. A. Freiwald, and D. Y. Tsao, "Patches with links: A unified system for processing faces in the macaque temporal lobe," Science, vol. 320, pp. 1355-1359, Jun. 2008.

[5] E. G. Ashby and N. A. Perrin, "Toward a unified theory of similarity and recognition," Physiol. Rev., vol. 95, pp. 124-150, 1988.

[6] S. L. Macknik, S. Martinez-Conde, and M. M. Haglund, "The role of spatiotemporal edges in visibility and visual masking," Proc. Nat. Acad. Sci., vol. 97, pp. 7556-7560, 2000.

[7] D. Bailey and R. Hodgson, "Range filters: Local intensity subrange filters and their properties," J. Image Vis. Comput., vol. 3, pp. 99-110, 1985.

[8] L.-L. Huang, A. Shimizua, Y. Hagiharab, and H. Kobatake, "Gradient feature extraction for classification-based face detection," Pattern Recognit., vol. 36, no. 11, pp. 2501-2511, Nov. 2003.

[9] A. Martinez and R. Benavente, The AR Face Database, CVC, 1998, Tech. Rep. 24.

[10] F. Samaria and A. Harter, "Parameterisation of a stochastic model for human face identification," in Proc. 2nd IEEE Workshop Applications of Computer Vision, Sarasota, FL, Dec. 1994.

[11] P. N. Belhumeur, J. P. Hespanha, and D. J. Kriegman, "Eigenfaces vs. Fisherfaces: Recognition using class specific linear projection," IEEE Trans. Pattern Anal. Mach. Intell., vol. 19, no. 7, pp. 711-720, 1997.

[12] C. V. Lab, Caltech Face Database, 1999. [Online]. Available: http:// www.vision.caltech.edu/html-files/archive.html.

[13] P. J. Phillips, H. Moon, P. J. Rauss, and S. Rizvi, "The FERET evaluation methodology for face recognition algorithms," IEEE Trans. Pattern Anal. Machine Intell., vol. 22, pp. 1090-1104, Oct. 2000.

[14] R. Beveridge, D. Bolme, M. Teixeira, and B. Draper, The CSU Face Identification Evaluation System Users Guide, Version 5.0, 2003. 